\title{
Das Gesicht der Sprache. Hugo von Hofmannsthals „Schlüssel-comödie“ Der Schwierige (1921)
}

„Anfang: ob es eine Schlüssel-comödie sei, also eine Infamie oder eine Persi"lage" - mit diesen Worten beginnt eine der beiden Notizen, in denen Hugo von Hofmannsthal im November 1921 einen Dialog mit dem Titel Kritik des „Schwierigen“ entwirft. „Die Dame, die behauptet, es sei eine Persiflage", so die Notiz weiter, „sagt auch es sei ein Ausfluss von snobism Kriecherei vor einer Welt die [...] gar nicht existiert...". . Hofmannsthal legt der fiktiven Dame hier eine Feststellung in den Mund, deren Provokation weniger in den Begriffen ,Infamie' oder ,Persiflage' selbst als vielmehr in einem von ihnen hervorgehobenen inneren Widerspruch besteht: Wenn die Welt, die angeblich von dem Schwierigen auf ehrenrührige Weise verunglimpft oder persifliert wird, gar nicht existiert', wie kann dann von jenem Abbildungs- oder gar Deutungsverhältnis zwischen dem Stück und dieser Welt die Rede sein, das der Begriff der ,Schlüssel-comödie' nahelegt?

Indem er diese Frage in den Blick rückt, reagiert Hofmannsthals Dialogentwurf darauf, in welch kontroverser Weise die ersten zeitgenössischen Rezipienten des Stückes seine Beziehung zur gegenwärtigen Wirklichkeit in den Mittelpunkt ihrer Reaktionen stellen. Stefan Großmann fasste es in seiner Rezension für das Tage-Buch als Komödie über die „komplizierte Inzucht" auf, an der die Donaumonarchie zugrunde gegangen sei. Erheblich harscher noch urteilte Julius Bab in der Hilfe nach einem Probenbesuch im August 1921, die Komödie sei „unüberzeugend, zufällig, flach, spielerisch“, weil ihr Bezug zur Nachkriegswirklichkeit fehle: „Hofmannsthals Milieu-Lustspiel spielt im leeren Zeitraum - eine Wiener Hocharistokratie nach dem Weltkrieg, die immer noch im erblichen Herrenhaus sitzt! Aber vielleicht hat die Wirklichkeit doch folgerichtiger gedichtet, als sie diese Herrenhäuser enterbte und zusammenbrechen ließ", weil, so die Implikation, die solcherart enterbte Kultur ohnehin nur künstlerisch Beliebiges, Unverbindliches hervorgebracht habe, und dieser Mangel an Verbindlichkeit sei, so Bab, „der Todespunkt, der dies ganze anmutreiche Spiel im leeren Zeitraum zu einem mehr als gebrechlichen Ge-

1 Hugo von Hofmannsthal, „Kritik des ,Schwierigen “", in: ders., Sämtliche Werke. Kritische Ausgabe, hg. v. Rudolf Hirsch, Christoph Perels, Edward Reichel u. Heinz Rölleke, Bd. 12, Dramen 10. Der Schwierige. Lustspiel in drei Akten, hg. v. Martin Stern in Zus.arb. m. Ingeborg Haase u. Roland Haltmeier, Frankfurt am Main, 1993, S. 514 f., hier S. 514. Zitatnachweise aus diesem Band werden im laufenden Text in Klammern direkt angeschlossen (in den Fußnoten mit der Sigle SW 12).

2 Stefan Großmann, o. T., in: Das Tage-Buch, 03.12.1921, zit. n. SW 12, S. 164-167, hier S. 166. 
schöpf macht."3 Raoul Auernheimer dagegen las den Schwierigen als „ein kulturhistorisch interessantes Stück Oesterreich, österreichischestes Oesterreich, im reinen Spiritus einer ungemein geistreichen Darstellung für kommende Zeiten aufbewahrt."4 Hofmannsthal dankte Auernheimer für "Geist und Wohlwollen" seiner Beurteilung, grenzte sich jedoch nachdrücklich von dem Gedanken eines sozialhistorischen Beispielpräparates ab: „Die Fundamente auf denen der Character des Schwierigen ruht", so Hofmannsthal in einer vielzitierten Passage seines Briefs, "suchen wir allerdings in sehr verschiedener Sphäre: Sie in der socialen, die mir doch mehr ein Secundäres erscheint, ich in einer tieferen: im schwierigen Verhältnis zur Rede und zur Tat, den für den Menschen verknüpfenden Magien". ${ }^{5}$

Als ,Schlüssel-comödie' im herkömmlichen Sinn eines Stücks, für dessen Protagonisten sich in der von der fiktiven Dame des oben erwähnten Dialogs beschworenen Welt personale Vorbilder identifizieren ließen, ist Der Schwierige in der Tat nicht gesamthaft, sondern allenfalls in einem Detail angelegt, das zudem von dem Stück selbst nicht explizit ausgespielt wird. In einem Brief vom 14. Oktober 1921 an Arthur Kahane, der als Dramaturg des Berliner Deutschen Theaters die Premiere der Komödie vorbereitete, antwortete Hofmannsthal auf eine Anfrage, die Kahane bezüglich des Bühnenbilds an ihn gerichtet hatte: „Photographie meines versperrten und verräumten Arbeitszimmers kann [ich] unmöglich beschaffen, sie wäre auch nicht von grossem Nutzen", da sich das Ambiente, in dem er arbeitete, aus Platzgründen nicht auf die Bühne bringen lasse: „Solche Zimmer sind sehr geräumig, geräumiger als auf der kleinen Bühne möglich" (511). Implizit konzedierte Hofmannsthal also einen konkreten Wirklichkeitsbezug, der freilich von der Figur Bühls weniger auf Kessler als vielmehr auf Hofmannsthal selbst verweist, und auch dies nicht anhand eines konkreten und als solchen wiedererkennbaren individuellen Signalements, sondern in poetologischer Referenz auf das Arbeitszimmer des Dichters, in dem die Komödie zu jener später so konfliktiv rezipierten Reflexion über das Wesen von Sprache geformt worden war.

Einig ist man sich in der Forschung schon längst, dass Hofmannsthals Schwieriger in dramatisch inszenierter Experimentalanordnung dieselbe Sprachkritik zum Gegenstand hat, die sein berühmter Chandos-Brief expliziert, ${ }^{6}$ und damit „das gelingende Werk" behauptet, das "gerade seiner Un-

3. Julius Bab, „Im leeren Zeitraum“, in: Die Hilfe, 15.08.1921, zit. n. SW 12, S. 155-159, hier S. $15^{8}$ f.

4 Raoul Auernheimer, „,Der Schwierige' von Hofmannsthal", in: Neue Freie Presse, 16.10.1921, S. 159-164, zit. n. SW 12, S. 512.

5 Hugo von Hofmannsthal u. Raoul Auernheimer, „The correspondence of Hugo von Hofmannsthal and Raoul Auernheimer", hg. v. Donald G. Daviau, in: Modern Austrian Literature 7 (1974), S. 209-307, zit. n. SW 12, S. 484.

6 Vgl. Richard Brinkmann, „Hofmannsthal und die Sprache", in: DVjs 35 (1961), S. 69-95; Emil Staiger, „Hugo von Hofmannsthal: ,Der Schwierige"', in: Hugo von Hofmannsthal, hg. v. Sibylle Bauer, Darmstadt, 1968, S. 402-433 Ehrhard Bahr, „Dezenz der Rede: Zur Sprachproblematik in Hofmannsthals Lustspiel ,Der Schwierige "', in: Austriaca. Beiträge zur österreichischen Literatur. Festschrift für Heinz Politzer zum 75. Geburtstag, hg. v. Winfried Kudszus u. 
entschiedenheit über Sprache sich verdanken soll“.7 Diese Experimentalanordnung aber legt eben nicht nur der handelnden Titelfigur die Resignation Lord Chandos' gegenüber dem ,schwierigen Verhältnis' zwischen ,der Rede und der Tat' in den Mund, sondern problematisiert darüber hinaus auch die logische Konsequenz aus dieser-Resignation. Auch die von Hans Karl Bühl gesprochenen Worte nämlich unterliegen diesem, schwierigen Verhältnis' insbesondere dann, wenn sie es zu fassen versuchen; auch er kann Rede und Tat nicht miteinander verknüpfen, wo diese Verknüpfung im Sog pervasiver Sprachkritik als unmöglich inszeniert wird: Die Feststellung des ,schwierigen Verhältnisses' ist immer auch eine rekursive Operation, muss immer auch auf sich selbst angewandt werden und zieht damit, wenn sie, wie in Hofmannsthals Komödie, Konstruktionsformel des gesamten Dramentextes ist, immer auch das in Zweifel, was die Operation selbst an Gewissheiten hervorzubringen scheint.

Kritiken wie diejenigen von Bab und Auemheimer, so gegensätzlich sie in ihrer Wertung auch sind, reagieren beide auf ein Problem, das sich aus diesem Sachverhalt ergibt. Eine Konstruktionsformel nämlich, die gleichsam a priori das im Stück Gesagte entmächtigt, und ein Stück, das den Prozess solcher Entmächtigung in poetischer und als solche sprachlich gestiftete Sinneinheit auf die Bühne zu bringen beansprucht, bilden einen auf den ersten Blick unvereinbaren Gegensatz: Entweder demontiert das auf der Bühne Gesagte den Realitätsbezug der Sprache konsequent und total, dann aber wird der im Stück geschaffene Spiel-Raum als gänzlich beliebiger zum ,leeren Zeitraum', wie ihn Bab beanstandet; oder aber diese Demontage selbst ist Bestandteil, ja zentrale Eigentümlichkeit einer Wirklichkeit, die sich darin als, so Auernheimer, ,österreichischestes Oesterreich ${ }^{`}$ wiederzuerkennen vermag. Die Wirklichkeit jedoch, auf die sich die Komödie bezieht, ist gerade nicht das von Hofmannsthal als ,Secundäres' deprivilegierte ,sociale', jene gesellschaftlich konventionalisierte und als solche intrinsisch theatralische Kommunikation der adligen Statusgruppe, die Auernheimer als ,österreichischestes Oesterreich' wiederzuer-

Hinrich C. Seeba, Tübingen, 1975, S. 284-297; Jürgen Rothenberg, „Durchs Reden kommt alles auf der Welt zustande. Hofmannsthals ,Der Schwierige“", in: Jahrbuch der Deutschen Schillergesellschaft. 21 (1977), S. 393-417; Axel Hübler, „Zur Konversation in Hugo von Hofmannsthals Der Schwierige", in: Literatur und Konversation. Sprachsoziologie und Pragmatik in der Literaturwissenschaft, hg. v. Ernest W. B. Hess-Lüttich, Wiesbaden, 1980, S. 115-130; Walter Pape, ",Ah, diese chronischen Mißverständnisse!' Hugo von Hofmannsthal: ,Der Schwierige"', in: Deutsche Komödien, hg. v. Winfried Freund, München, 1988, S. 209-225; Klaus-Dieter Krabiel, „Sprachskepsis im Konversationsstück? Hugo von Hofmannsthals Lustspiel ,Der Schwierige'", in: Verbergendes Enthüllen. Zur Theorie und Kunst dichterischen Verstehens. Festschrift für Martin Stern, hg. v. Wolfram Malte Fues u. Wolfram Mauser, Würzburg, 1995, S. 311-328.

7 Helmut Arntzen, „Der Schwierige und der Nörgler. Sprecherphysiognomien und Sprachreflexion in Hofmannsthals Nachkriegslustspiel und Karl Kraus' Weltkriegstragödie", in: Physiognomie und Pathognomie. Zur literarischen Darstellung von Individualität. Festschrift für Karl Pestalozzi zum 65. Geburtstag, hg. v. Wolfram Groddeck u. Ulrich Stadler, Berlin/New York, 1994, S. 344-359, hier S. 359. 
kennen glaubt. Vielmehr bildet Hofmannsthal das Wesen einer Rede; die in virtuoser Geschliffenheit von der Unmöglichkeit gelingenden Sprechens spricht, als Inbegriff einer fundamental paradoxen conditio humana ab: Weil der Mensch der Sprache mächtig ist, kann er über deren ,schwieriges Verhältnis' zur magisch anmutenden Verknüpfung von Rede und Tat zwar sprechen, sich dieser Verknüpfung letztlich aber nicht entziehen, gleich welche „Missverständnisse" (13) - einer der Leitbegriffe der Komödie - er sprachlich erzeugt, um den Unterhaltungswert der Komödie als Wert fortgesetzt irregehender Unterhaltungen zu konstituieren.

So sind es nicht diese irregehenden Unterhaltungen an sich, die der Komödie ihre Struktur und damit auch ihren Wirklichkeitsbezug geben, sondern ein Komplex aus drei in den Dialogen wie in Regieanweisungen wiederkehrenden Leitmotiven: den Motiven des Schlüssels, ${ }^{8}$ der Brücke und des Gesichts. In einer - in die Endfassung nicht aufgenommenen - Notiz aus dem Jahr 1917 zur ersten Szene der Komödie wird die Bedeutung dieser Motive für die Entwicklung der Figur Hans Karl Bühls von dem Diener Lukas gegenüber dem neuen Diener Vinzenz mit der Formulierung eingeführt, man müsse „[a]uf solche kleine Zeichen [...] bei ihm gut achten, denn aus dem was er zu ihnen sagt werden sie nicht wissen woran sie mit ihm sind". (310). Gemeint ist hier zunächst Bühls Umgang mit Schlüsseln, jenem Motiv, das den ersten Akt dominiert und damit sowohl diesen Akt als Schlüssel zu den folgenden beiden Akten ausstellt als auch den Begriff der ,Schlüssel-comödie‘ als Bezeichnung für eine Komödie lesbar macht, die von Schlüsseln handelt. Als rezeptionsästhetischer Hinweis aber umfasst diese Formulierung mit dem Schlüssel zugleich auch die Motive der Brücke und des Gesichts. Das der Brücke wird im zweiten Akt eingeführt, wo es sowohl den Akt selbst als Brücke zwischen dem ersten und dem dritten Akt kennzeichnet als auch das Wesen der inszenierten Rede aus der Perspektive auf deren Brückenfunktion durchspielt; das Motiv des Gesichts dagegen durchzieht die gesamte Komödie in jenem doppelten Sinn von Antlitz und Vision, in dem am Ende darin das Wesen der Sprache selbst sichtbar wird. ,Klein' sind diese Zeichen ihrerseits in doppeltem Sinn: Zum einen werden sie nicht oder nur marginal handlungsrelevant - was ihren poetologisch-selbstreferentiellen Charakter hervorhebt -; zum anderen stehen sie in ihrer Bedeutungsführung diskret, aber beharrlich quer zum Strom der großen Zeichen der hochelaborierten Figurenrede und konterkarieren diese so durch eine Art medialen Meta-Raums, in dem und durch den Bühl nicht nur eminent kommunikationsfähig ist, sondern auch zur Verknüpfung von Rede und Tat findet.

8 Vgl. dazu Manfred Misch, „Der Dietrich der Seele. Zur Sprachproblematik in Hofmannsthals ,Der Schwierige“", in: Festschrift für Herbert Kolb zu seinem 65. Geburtstag, hg. v. Klaus Matzel u. Hans-Gert Roloff unter Mitarb. v. Barbara Haupt u. Hilkert Weddige, Bern u. a., 1989 , S. $483-493$. 
„Der Anfang entschleiert die Gebärde des Schreibenden", 9 notiert Hofmannsthal 19o9, in dem Jahr also, in dem die ersten Entwürfe zum Schwierigen entstehen. Bezieht man diese Überlegung auf den ersten Satz der Figurenrede in der Komödie, so ,entschleiert' dieser die ,Gebärde des Schreibenden' als eine, die auf Sprachhandlungen weist: „Hier ist das sogenannte Arbeitszimmer", erklärt der alte Diener Lukas dem probeweise eingestellten neuen Diener Vinzenz und fügt hinzu: „Verwandtschaft und sehr gute Freunde werden hier hereingeführt oder, nur wenn speziell gesagt wird, in den grünen Salon." (7) Die erste zusammenhängende Niederschrift des Schwierigen von 1917 hat nach dem Wort ,Arbeitszimmer" noch den Satz: „Früher hat es geheissen der weisse Salon, noch früher bei Erlaucht Graf Leopold war es Sommerspeiszimmer." (305) Die gewissermaßen autorlosen Formulierungen dieses Satzes - ,es hat geheissen', ,es war' - bezeugen, dass es Hofmannsthal darum zu tun war, von Anfang an die Bedeutung der gräflichen Sprachhandlungen hervorzuheben, hier zunächst solche Sprachhandlungen, die im durch die Standesprivilegien reichen Erbadels abgesicherten Modus der autoritativen Anweisung - des Nennens und ,speziell Sagens - Rede und Tat mit scheinbar unhinterfragbarer Selbstverständlichkeit zu verknüpfen in der Lage sind. Das Weglassen dieses Satzes, zusammen mit der Streichung des in dieser Niederschrift unmittelbar folgenden Satzes "Hier ist sein Schreibtisch an dem er arbeitet" (ebd.), fokussiert diese Bedeutung in einer betont paradoxen ${ }^{10}$ Wendung, die den Modus gelingenden Nennens bereits im ersten Satz der Komödie in Zweifel zieht: Das Arbeitszimmer, eigentlich Raum der solitären schriftlichen Sprachhandlung, erfüllt zugleich die soziale Funktion eines Empfangsraums für Freunde und Verwandtschaft und trägt damit den Namen einer Tätigkeit, die durch diese Funktion strukturell der fortgesetzten Störung unterliegt.

Von den ersten Notizen zum Schwierigen an ist Brühls Verhältnis zur Beziehung zwischen Sprache und Realität als zutiefst problematisch angelegt. „Hans Karl zweifelt an dem Festen Gegebenen", heißt es in einer Notiz vom 13.01.1910: „Die Unterschiede, die couranten Unterschiede zwischen den Menschen, auch die couranten Wertungen sind ihm abhanden gekommen. (vergl. ,ein Brief')" (223). Und noch 1921 schreibt Hofmannsthal an den Direktor des Burgtheaters Anton Wildgans über den Schwierigen: „Es ist das Problem das mich oft gequält u. beängstigt hat ([...] am stärksten in dem ,Brief' des Lord Chandos, den Sie vielleicht kennen) - wie kommt das einsame Individuum dazu, sich durch die Sprache mit der Gesellschaft zu verknüpfen, ja durch sie, ob es will oder nicht,

9 Hugo von Hofmannsthal, Gesammelte Werke in zehn Einzelbänden, Bd. 10, Reden und Aufsätze III (1925-1929). Aufzeichnungen, hg. v. Bernd Schoeller u. Ingeborg Beyer Ahlert, Frankfurt am Main, 1980, S. 499.

10 Zur Bedeutung des Paradoxen für die Anlage der Komödie vgl. Ralf Simon, „Paradoxien der Interpretation (ausgehend von Hofmannsthals ,Der Schwierige')“, in: Jahrbuch der Deutschen Schillergesellschaft 46 (2002), S. 199-218. 
rettungslos mit ihr verknüpft zu sein?"11 Dem 1902 verfassten Brief ist mit der Darstellung, die Lord Chandos darin von der verlorenen Unschuld seines Selbstbewusstseins als Autor gibt, eine Antwort auf diese Frage bereits mit eingeschrieben - und es ist diese Antwort, in der das Motiv des Schlüssels im Schwierigen vorgeprägt ist: „[E]s ahnte mir", erinnert sich Chandos, „alles wäre Gleichnis und jede Kreatur ein Schlüssel der andern, und ich fühlte mich wohl den, der imstande wäre, eine nach der andern bei der Krone zu packen und mit ihr so viele aufzusperren, als sie aufsperren könnte. ${ }^{\text {"12. }}$

Die Erkenntnis Chandos', dass ein solches Vertrauen auf die Realitätstauglichkeit sprachlich deutender Sinnstiftung nur in die Irre führen kann, ist der Figur des Hans Karl Brühl von Beginn an eingeschrieben. Im Gegensatz zu seiner Schwester, die durchweg auf der klärenden Macht der Rede beharrt, ist die verbale Kommunikation für Hans Karl eine Fessel, zu deren Öffnung es ihm an einem Schlüssel mangelt, und weil sie eine solche Fessel ist, ist sie zugleich immer auch der Ort, an dem katastrophisch und unabwendbar Unordnung hereinbricht: „Er wird“, so die Notiz weiter, „von allen der Confident, aber dieses [...] Verhältniss verhäkelt sich bei ihm mit jedem. Er führt ein Gespräch, um jemanden zu überreden, zu beruhigen, zu entfernen - und verwirrt, beunruhigt, verbandelt den Betreffenden" (221). Selbst gefesselt - , verhäkelt ${ }^{\prime}$ - im Gefüge eines Mediums, dessen semantische Unschärfen immer neue Ausdeutungen und damit immer neue Projektionspotentiale wie die eröffnet, die Antoinette Hechingen in ihrem Bemühen realisiert, die Trennung abzuwenden, ,verbandelt ' Hans Karl seine Dialogpartner umso unausweichlicher, als er gerade deshalb; weil er an der Sprache zweifelt, von allen anderen als Inkarnation der Ehrlichkeit und Verlässlichkeit wahrgenommen wird: Wer ständig sagt, dass er nicht sagen kann, was er sagen möchte, erweckt den Eindruck, als wisse er besonders gut, was eigentlich zu sagen wäre.

Dieses fatale double bind führt bei Hans Karl zu einem geradezu obsessiven gestischen Bemühen um eben die Ordnung, die er sprachlich permanent verfehlt, einem Bemühen, das der erste Akt vor allem am Motiv des Schlüssels ausspielt. Kurz nachdem der alte Diener Lukas den neuen Diener Vinzenz in das ,sogenannte Arbeitszimmer' des Grafen eingeführt hat, erläutert er ihm mit den folgenden Worten einige von dessen Eigenheiten: „Er kann kein Bild und keinen Spiegel schief hängen sehen. Wenn er anfängt, alle Laden aufzusperren oder einen verlegten Schlüssel zu suchen, dann ist er schlechter Laune." (8) Während wenig später Hans Karls Schwester Crescence zu ihm von ihren früheren Hoffnungen auf eine Verbindung zwischen ihm und Helene Altenwyl spricht, löst Bühls Gestik Lukas’ Worte ein, zieht „eine Lade am Schreib-

11 Hugo von Hofmannsthal - Anton Wildgans, Briefwechsel, hg. und kommentiert von Norbert Altenhofer, Heidelberg, 1971, S. 31.

12 Hugo von Hofmannsthal, „Ein Brief“, in: ders., Sämtliche Werke. Kritische Ausgabe, hg. v. Rudolf Hirsch, Christoph Perels, Edward Reichel u. Heinz, Rölleke, Bd. 31, Erfundene Gespräche und Briefe, hg. v. Ellen Ritter, Frankfurt am Main, 1992, S. 45-55, hier S. 48. 
tisch heraus“, „sucht in der Lade nach etwas“, „stösst die Lade zu“, „zieht mit einiger Heftigkeit eine andere Lade heraus" und erklärt schließlich auf die Frage seiner Schwester, was er suche: „[I]ch such' eigentlich gar nichts, ich hab' den falschen Schlüssel hineingesteckt." (14 f.) - eine Aussage, die nur dann eine Antwort auf Crescences Frage ist, wenn das, was Bühl tatsächlich gesucht hat, der passende Schlüssel zum Verständnis der von dem Gesprächsthema ausgelösten ,schlechten Laune' ist.

In den Entwürfen zum Schwierigen sind mehrere Varianten zu Lukas' Part überliefert, die dessen Bedeutung weiter erhellen. Wenn er „schlechter Laune ist", erklärt Lukas in der Niederschrift vom September 1917, „richtet er Bilder oder er verlegt einen Schlüssel oder sperrt mit falschem Schlüssel auf - diese kl. Zeichen muss man bei ihm gut beachten, weil er im Ausdruck behindert ist" (306). In der oben bereits zitierten späteren Notiz aus derselben Arbeitsphase schwächt Hofmannsthal den letzten Teil des Satzes ab: „Auf solche kleine Zeichen muss man bei ihm gut achten, denn aus dem was er zu ihnen sagt werden sie nicht wissen woran sie mit ihm sind, so eine directe Äusserung liebt er nicht" (310). Allein Lukas kann die ,kleinen Zeichen' richtig lesen und ist so im Besitz des oder zumindest eines richtigen Schlüssels - ein Tatbestand, den Hofmannsthal in der Endfassung dadurch unterstreicht, dass er in der letzten Szene dieses ersten Aktes Lukas per Regieanweisung ein Zigarrenkästchen zuschließen lässt und damit das erste und einzige Mal im Stück überhaupt einen richtigen Schlüssel ins Spiel bringt. Im Spiel mit dem Schlüssel enthüllt sich das Stück in dieser Szene buchstäblich als "Schlüssel-comödie“ - eine Komödie, die in dem Maß, in dem sie den Schlüssel im Wortsinn einsetzt, gerade nicht im Sinne von ,Infamie' oder ,Persiflage' auf eine gesellschaftliche Wirklichkeit außerhalb des Stückes zielt, sondern die vielmehr mit dem Motiv des Schlüssels die Möglichkeit sprachlicher Sinnstiftung schlechthin codiert. Die Zuordnung des richtigen Schlüssels zu der Figur des Lukas öffnet dabei eine sozialhistorische Dimension: Die Sprachhandlungen des Grafen erreichen ihren Zweck nur dann störungsfrei, wenn sie durch das unumkehrbare hierarchische Gefälle einer spätfeudalistischen Herr-Domestiken-Beziehung abgesichert sind, in der, wie übrigens auch in Hofmannsthals ähnlich gebauter KomödieDerUnbestechliche, derDienerbuchstäblichdieSchlüsselgewalthat - dies zumindest suggeriert die Beziehung zwischen Brühl und Lukas. Im Kontext der Komödie aber reicht diese Absicherung bereits nicht mehr aus, um die von Bühl so vielfach beklagten Missverständnisse auch nur zu kontrollieren, geschweige denn zu verhindern. Die Gespräche des Grafen mit Familienmitgliedern und mehr noch die mit Mitgliedern des gesellschaftlichen Kreises, der sich abends im Altenwyl'schen Salon zusammenfinden wird, sind schon im ersten Akt durch solche Missverständnisse geprägt, eine Serie permanenter Störungen im Kommunikationsprozess, die in Bühls Telefongespräch mit seinem Freund und dem Gatten seiner ehemaligen Geliebten Antoinette Hechingen kulminieren: Die Verbindung ist so schlecht, dass die mehrfache Wiederholung der Aussage, Hechingens Versöhnung mil Antoinette entspreche auch 
seiner, Bühls, „Empfindung“, schließlich dazu führt, dass Bühl sie als „gleichgültige Phrase" bezeichnet, "weil du es so lange nicht verstanden hast" (53).

Dergestalt in seiner Bedeutung systematisch performativ ausgehöhlt, verschwindet der Schlüssel, der am Anfang des Akts als Hoffnungsträger für die Möglichkeit einer - wiewohl auf dem Umweg über ,kleine Zeichen - gelingenden Kommunikation eingeführt worden ist, an dessen Ende aus dem Stück. Abgelöst wird er durch das noch im Telefongespräch mit Hechingen eingeführte Motiv der Brücke, das darin als Name des Bridge-Spiels auftaucht: Da „ich nicht Bridge spiele und Deine Frau, wie ich glaube, auch nicht" (52), sieht Bühl in der kommenden Soiree die passende Gelegenheit, durch eine „ernste Konversation mit der Toinette" eine Brücke zwischen den entfremdeten Eheleuten zu schlagen und zudem im Auftrag seiner Schwester "die gewissen seriosen Unterhaltungen" (6o) mit Helene und ihrem Vater zu führen, aus denen die Verlobung der ersteren mit Stani hervorgehen soll. Der Grund für die Bereitwilligkeit, mit der Bühl solcherart als Familienvorstand bei der bevorstehenden Soiree „ein richtiges Programm“ (61) zu absolvieren plant, ist, so Hofmannsthals Zusammenfassung des ersten Aktes für die Neue Freie Presse, die Hoffnung des Grafen auf endgültige Ablösung seiner Verpflichtungen: „[I]ndem er zwei glückliche Paare schafft, wird er zugleich, so schwebt's ihm vor, die letzten Brücken abbrechen, die ihn selbst mit Jugend und Leben verbinden. ${ }^{13}$

Wie im ersten Akt; so enthalten auch im zweiten die erste Regieanweisung und der erste gesprochene Satz programmatische Informationen. „Bei Altenwyls. Kleiner Salon im Geschmack des XVIII. Jahrhunderts", lautet die Regieanweisung, und Altenwyl begrüßt Graf Bühl mit den Worten: „[I]ch rechne dir dein Kommen doppelt hoch an, weil du nicht Bridge spielst und also mit den bescheidenen Fragmenten von Unterhaltung vorlieb nehmen willst, die heutzutage in einem Salon noch geboten werden." (63) Dass es selbst dann, wenn man die Wortwahl Hofmannsthals in der eben zitierten Zusammenfassung des ersten Akts außer Acht lässt, ganz und gar kein Zufall ist, dass an diesem Abend ausgerechnet Bridge gespielt wird, geht aus der unmittelbar anschließenden Einmischung Crescences und der Reaktion Altenwyls hervor: „[I]ch hab drüben an meinem Bridgetisch ein ganz neues Gesicht", bemerkt Crescence, und Altenwyl erklärt daraufhin, es handle sich dabei um einen „liebe[n] politische[n] Kollege[n]“, „Professor Brücke“ (ebd.).

Mit dem Motiv der Brücke verleiht Hofmannsthal dem Schauplatz Salon diskret das cachet des theatrum mundi. Bereits in der wohl frühesten Ausformung des Welttheatertopos in seinem Werk, dem Einakter Das kleine Welttheater von 1897, hatte dieses Motiv eine zentrale Funktion übernommen: „Die Bühne", so die Regieanweisung, „stellt den Längsschnitt einer Brücke dar, einer

13 Hugo von Hofmannsthal, o. T., in: Neue Freie Presse, 25.07.1920, S. 1, zit. n. SW 12, S. 493 f., hier S. 494 . 
gewölbten Brücke, so dass die Mitte höher liegt als links und rechts. ${ }^{\text {“14 }}$ Im Welttheater des Altenwyl'schen Salons markieren das Kartenspiel und der Professor mit dem sprechenden Namen die Außenpositionen des Spektrums von Möglichkeiten, in dem sich die Sprachhandlungen der Salongäste bewegen am einen Ende das hochkomplizierte Strategiespiel einer müßigen Elite, am anderen die humorlos selbstverliebte Sprachkompetenz des bürgerlich-bornierten Akademikers, der auf der Suche nach Akzeptanz durch den Adel immer wieder gerade dann in die Gespräche Bühls gerät, wenn diese Gespräche ohne seine Anwesenheit zu gelingender Kommunikation hätten führen können. Die Brückenfunktion mithin, die die verbale Kommunikation übernehmen könnte, wird von eben jener unheilbaren Entfremdung zwischen Bezeichnendem und Bezeichnetem sabotiert, die Hofmannsthals Chandos einst sein Vertrauen in die Möglichkeit sprachlicher Sinnstiftung hatte verlieren lassen: Das Kartenspiel erzeugt Segregation statt Verknüpfung, indem es die Gruppe der Spielenden von den anderen Gästen isoliert, und der professorale Autor zerstört zwischen Bühl und Helene permanent genau das, was seinem Namen als Auftrag eingeschrieben ist.

Der Ursprung dieser Zerstörungskraft wird von dem Stück dem Ersten Weltkrieg zugewiesen. Es spiele, schreibt Hofmannsthal am 14. Juli 1918 an den Frankfurter Theaterdirektor Karl Zeiss, „in der unmittelbaren Gegenwart mehr als das: es setzt den Krieg als beendet voraus, die handelnden Figuren, soweit sie Männer sind, waren im Kriege, diese Vergangenheit ist Voraussetzung . ${ }^{15}$ Für Bühl ist er unmittelbare Voraussetzung dafür, am Ende zur Verlobung mit Helene zu gelangen - erst allerdings, nachdem er seine Zerstörungskraft dazu eingesetzt hat, in seinem Gespräch mit Helene in der 14. Szene des zweiten Akts die Brücke zu ihr vordergründig noch wesentlich konsequenter zu zerstören, als dies dem gleichnamigen Professor gelingt. Ansatzpunkt dafür ist die von ihm heraufbeschworene Erinnerung an „dieses Verschüttetwerden" (101), eine Erfahrung, deren konkreten geschichtlichen Kontext das Gespräch mit Helene ostentativ verschweigt. ${ }^{16}$ Angesichts der Bedeutung, die das Motiv der Brücke für den zweiten Akt besitzt, legt dieses Verschweigen die Vermutung nahe, Bühl könne im Krieg bei der Sprengung einer Brücke ver-

14 Hugo von Hofmannsthal, „Das kleine Welttheater", in: ders., Sämtliche Werke. Kritische Ausgabe, hg. v. Rudolf Hirsch, Christoph Perels, Edward Reichel u. Heinz Rölleke, Bd. 3, Dramen 1, hg. v. Götz E. Hübner u. Klaus E. Pott, Frankfurt am Main, 1982, S. 131-149, hier S. 133.

15 Cynthia Walk, „.... in der unmittelbaren Gegenwart'. Hugo von Hofmannsthals Briefe an Karl Zeiss", in: Hofmannsthal-Blätter 30 (1984), S. 56-68, hier S. 64.

16 Zu Einordnung und Deutung dieser Erfahrung im Kontext des Ersten Weltkriegs insbesondere aus psychologischer Perspektive vgl. Inka Mülder-Bach, „Herrenlose Häuser. Das Trauma der Verschüttung und die Passage der Sprache in Hofmannsthals Komödie ,Der Schwierige"', in: Hofmannsthal-Jahrbuch 9 (2001), S. 137-161, sowie Maximilian Bergengruen, „,Mystik der Nerven': Neurasthenie, Zerstreutheit und die Metaphysik des Willens in Hofmannsthals ,Der Schwierige"', in: DVjs 80 (2006), S. 212-244, und Hans Hahn, „Intertextuelle Studien zur Kriegsheimkehr: Ein heuristischer Versuch", in: German Life and Letters 67 (2014), S. 341-357. 
schüttet worden sein; wäre dies der Fall, dann wäre das Gespräch mit Helene über ,dieses Verschüttetwerden' eine kathartische Wiederholung des dabei erlittenen Traumas, die, indem sie die Ursache für Bühls melancholische Überzeugung vom Misslingen jeglicher verbaler Kommunikation freilegt, gewissermaßen den Weg für die finale Verständigung der beiden Liebenden im dritten Akt freisprengt. ${ }^{17}$

Die Vermutung, dass dies so sein könnte, wird davon gestützt, dass in diesem Gespräch das Motiv des Gesichts und damit jenes poetologische Element, in dessen Zeichen die Verlobung schließlich stattfinden wird, betont in den Vordergrund gerückt wird. Gesichter sind in der Komödie von Anfang an Gegenstand der Einzeichnung und des Ablesens von - in den meisten Fällen entweder falsch entzifferten oder von dem Lesenden projizierten - Bedeutungen. „Ihm steht's doch auf der Stirne geschrieben, daß er erreicht, was er sich vornimmt" (58), kommentiert Bühl gegen Ende des ersten Akts die Erfolgsaussichten seines zur Werbung um Helene entschlossenen Neffen Stani, eine Vorhersage, die sich am Ende des Stückes als Fehllektüre erweist. Dennoch, oder vielmehr gerade deswegen, besitzt Bühls Lektüre von Stanis Gesicht eine kaum verborgene Tiefendimension, kraft derer sie präzise beschreibt, was er in diesem Moment in Stani sieht: Das Stani ,auf der Stirn' Eingeschriebene spielt auf das Kainsmal an, mit dem Kain dem Alten Testament nach von Gott auf der Stirn als Brudermörder gezeichnet worden ist, und zeigt Stani mithin in einer Drastik als Rivalen um die Gunst Helenes, mit der Bühls eigene Gefühle für letztere offensichtlich werden.

Was Bühl mithin in den von ihm gedeuteten Gesichtern liest, ist nicht das Antlitz im Sinne einer physischen Repräsentation der Eigenschaften und Befindlichkeiten der jeweiligen Person, sondern Gesicht im Sinne seiner eigenen Vision dessen, was diese Person für ihn bedeutet, und damit ein Kommunikationsakt. Der sprachliche Ausdruck, den er diesen Lektüren gibt, ringt um eben jene „letzte unaussprechliche Nuance“, die er zu Beginn seines Gesprächs mit Helene als „durch wohlgesetzte Worte" (97) nicht erreichbares Kommunikationsziel beschreibt. Wenn er Helene sagt, sie sei ,ja unzerstörbar, das steht ja deutlich in Ihrem Gesicht geschrieben“ (99), spricht er zwar vordergründig davon, dass sie einen anderen Mann heiraten solle als ihn, trägt ihr aber faktisch an, durch ihre eigene Unzerstörbarkeit die Spuren zu tilgen, die die Zerstörungsmacht des Krieges in ihm hinterlassen hat. Dass er, wie er ihr erläutert, „draußen begreifen gelernt habe: daß in den Gesichtern der Menschen etwas geschrieben steht" (100), ist eine dieser Spuren, hat doch die traumatische Erfahrung der Kriegsgewalt allen Menschen, die er ,draußen' erlebt hat, das

17 Die Figur der Wiederholung, wie sie bereits im Telefongespräch zwischen Bühl und Hechingen zu Ende des ersten Akts erschienen ist, ist ein Charakteristikum des klassischen Melancholikers, vgl. Ulrich Breuer, „Semper eadem'. Melancholie als Wiederholungszwang", in: Kommunikative Routinen. Formen, Formeln, Forschungsbereiche. Festschrift für Irma Hyvärinen zum $6_{5}$. Geburtstag, hg. v. Leena Kolehmainen, Hartmut Lenk u. Liisa Tiittula, Frankfurt am Main u. a., 2014, S. 135-151. 
Kainsmal des Brudermörders eingeschrieben, das er im ersten Akt mit Stani in Verbindung gebracht hat. Das „Notwendige“ (ebd.), als dessen Ausdruck er Helenes Gesicht liest, meint jene authentische Verknüpfung von Sein und Scheinen, Antlitz und Vision, die ihm durch , dieses Verschüttetwerden' abhandengekommen ist. Seine Erzählung davon erlaubt es ihm nun, diese Verknüpfung wiederherzustellen, indem er ihr berichten kann, er habe während der dreißig Sekunden, in denen er verschüttet lag, eine "ganze Lebenszeit [...] gelebt [...], und in diesem Stück Leben, da waren Sie meine Frau ${ }^{\text {"18 }}$ - und habe daraufhin während seines folgenden Spitalaufenthaltes ihr Gesicht bei der Eheschließung imaginiert: "[I]ch hab mir das in einer ganz genauen Weise vorstellen können, wie das alles sein wird, [...] wie sich's gehört zu Ihren Augen und zu Ihrer Stirn und zu Ihren Lippen, die nichts Überflüssiges reden können" (103).

Helene ist vielleicht von allen Figuren des Stückes diejenige, die Hofmannsthal im Laufe seiner Arbeit der tiefgreifendsten Wandlung unterwirft, um das Resultat eines in ihrem Gesicht geschriebenen ,Notwendigen' und damit die Idee einer Authentizität hervorzubringen, deren Wesen und Erscheinung miteinander identisch sind. "Herz wer weiss was das ist - wir sind schwache Tiere" (407), seufzt Helene in Bühls Armen nach dem Verlobungskuss noch in der Arbeitsschicht von 1918/19, in der Bühl mit Helene noch eine weibliche Sexualität entgegentritt, die nicht nur elementar gezeichnet ist, sondern sich explizit elementar weiß: „Alles was Sie durchmachen ist nichts. Aber wo ich hinabtauche dort ist etwas: dort geht's ins lebendige Fleisch. Dort gibts nicht Scham und schamlos - ah nein!", antwortet sie auf seinen Bericht von seinen Kriegserfahrungen, um dann ihrerseits in ihm das elementar Männliche zu beschwören: „Du kannst verführen ich weiss wie und nicht bloss den Mund suchst du sondern das Ganze“; „So ein Mann! / [...] - wenn meine Hüften breiter wären oder meine Fesseln gröber so wäre er dahin und verloren" (4O7 f.).

Die erotische Dimension dieser früheren Entwürfe wird von Hofmannsthal für die Endfassung so radikal wie systematisch zugunsten des ,Notwendigen getilgt, mit dem Bühl in Helene die Wiederherstellung seines, schwierigen Verhältnisses zur Rede und zur Tat, den beiden die Menschen verknüpfenden Magien', begehrt: Helenes Gesicht ist dasjenige einer Sprache, die diese Wiederherstellung zu leisten vermag - und damit zugleich dasjenige der Nation, aus deren Schicksal während und nach dem Ersten Weltkrieg Hofmannsthal seine Identität als durch Sprache handelnder Dichter neu zu begründen suchte. In seiner Ankündigung einer Österreichischen Bibliothek von 1915 setzt Hofmannsthal in diesem Zusammenhang den Begriff des Gesichts dazu ein, diese Identität visionär sichtbar zu machen: „Österreich hat in diesen Tagen seine Kraft gezeigt und vor der Welt wieder offenbar gemacht, daß es ein Wesen ist [...]. Es ist, als ob dieses Wesen alle hundert Jahre einmal sein Gesicht zeigen dürfe,

18 Vgl. dazu Breuers Feststellung, dass der Wiederholungszwang des Melancholikers, wie Bühl ihn an den Tag legt, „als Folge eines defizitären Zeitverhältnisses verstanden werden“ muss (Breuer, „Semper eadem'. Melancholie als Wiederholungszwang", S. 136). 
dann bleibt an dieses schöne Angesicht nur eine dumpfe Erinnerung die sich

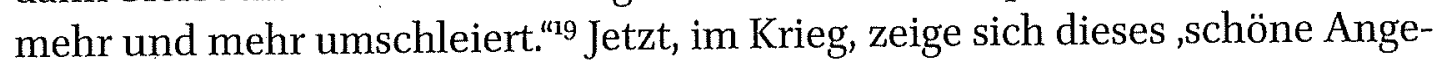
sicht' - so, wie Helenes Gesicht sich Bühl während seiner Verschüttung gezeigt hat: „[E]s muß wieder und wieder einem bösen, finsteren Geist, der uns niederhält, mit einer Schüssel Blut abgekauft werden. Es ist, als ob ein Aderlaß immer wieder uns den Kopf freimachen müßte, daß wir erkennen und lieben können. Ein solcher ist über uns gekommen, nun ist Österreichs Antlitz für uns wieder hervorgetreten."20

Wie Bühl an Helenes Gesicht ihre Unzerstörbarkeit in einem Moment beschwört, in dem Helenes Körpersprache ${ }^{21}$ ihre faktische Zerstörbarkeit signalisiert - sie ist während seiner Erzählung laut Regieanweisung „dem Umsinken nahe, beherrscht sich aber" (103) -, so formuliert auch Hofmannsthal seine Vision von ,Österreichs Antlitz' zu einem Zeitpunkt, zu dem er die Realitätshaltigkeit dieser Vision längst anzuzweifeln begonnen hatte. Schon im August 1913, anlässlich einer der Krisen auf dem Balkan, die dem Krieg vorausgingen, schreibt Hofmannsthal an Leopold von Andrian, er habe

das Vertrauen vor dem obersten Stand, dem hohen Adel, das ich hatte, das Zutrauen, er habe, gerade in Österreich, etwas zu geben und zu bedeuten, völlig verloren, und damit meine Achtung vor dem Stand als solchem [...]. Wir müssen es uns eingestehen, Poldy, wir haben eine Heimat, aber kein Vaterland - an dessen Stelle nur ein Gespenst. ${ }^{22}$

Zwar begrüßte Hofmannsthal den Kriegsausbruch im Sommer 1914 zunächst mit einer ähnlichen Euphorie wie viele andere zeitgenössische Intellektuelle auch und bezog eine Zeitlang durchaus propagandistisch Stellung für Österreich im Krieg. Während des Kriegs aber begann die welt- eine lebensgeschichtliche Krise in ihm auszulösen, an deren Ende Hofmannsthal, Österreichs Antlitz' statt in demjenigen einer zum,Gespenst' gewordenen politischen Großmacht nun in der Vision einer Kulturnation suchte.

Die Arbeit an seinem poetischen Werk gewann damit die Bedeutung einer zunächst höchst ambivalenten Auseinandersetzung mit seiner eigenen politi-

19 Hugo von Hofmannsthal, Sämtliche Werke. Kritische Ausgabe, hg. v. Rudolf Hirsch, Christoph Perels, Edward Reichel u. Heinz Rölleke, Bd. 36, Herausgebertätigkeit, hg. v. Donata Miehe, Catherine Schlaud, Ellen Ritter und Katja Kaluga, Frankfurt am Main, 2017, S. 27-33, hier S. 28.

20 Ebd.

21 Zur Bedeutung der Körpersprache in Der Schwierige vgl. Gerhard Austin, Die Phänomenologie der Gebärde bei Hugo von Hofmannsthal, Heidelberg, 1981, bes. S. 228-237; Thomas Heine, „The Force of Gestures: A New Approach to the Problem of Communication in Hofmannsthal's ,Der Schwierige“", in: German Quarterty 56 (1983), S. 408-418; Peter Kofler, „,Der Schwieriget von Hugo von Hofmannsthal: Die Geburt der Komödie aus der Gebärde der Melancholie“, in: Geste und Gebärde. Beiträge zu Text und Kultur der Klassischen Moderne, hg. v. Isolde Schiffermüller, Innsbruck/Wien/München, 2001, S. 158-188.

22 Hugo von Hofmannsthal u. Leopold von Andrian, Briefwechsel, hg. v. Walter II. Perl, Frankfurt am Main, 1968, S. 199 f. 
schen als dichterischen Identität, deren Ausgang Hofmannsthal bei Kriegsende Andrian gegenüber resümiert: "[I]ch kann sagen daß ich in diesen furchtbaren Jahren verstehen gelernt habe, was ein dichterisch veranlagtes Individuum bedeutet und daß ich mich zu diesen zu zählen habe - kurz meiner Vocation sicher geworden bin". ${ }^{23}$ Was das hieß, notierte im selben Jahr Berta Zuckerkandl, eine der berühmten Salonnieren des zeitgenössischen Wien, nach einem Besuch Hofmannsthals: „Alles Leid, der tiefe Gram, den er, der sein Land über alles liebte, in sich verschloß, wurde ihm Vision und Wille: das österreichische Gesamtkunstwerk für die Welt erstehen zu lassen". ${ }^{24}$ Wie Bühl in der Vision von Helenes Gesicht bei ihrer Trauung zu jenem Begehren findet, das am Ende der Komödie die Möglichkeit schafft, diese Vision in die Realität einer Trauung mit ihr überführen zu können, so findet Hofmannsthal in der Vision von Österreich als Kulturnation das ,Notwendiget seiner ,Vocation': das Gesicht einer Sprache, die sprachliche Kommunikation gerade dadurch gelingen lässt, dass sie von der Unmöglichkeit gelingenden Sprechens spricht.

Der letzte Satz des Schwierigen bringt dieses Gesicht noch einmal in dieser für die Sprachhandlung der Komödie so charakteristischen paradoxen Konfiguration auf die Bühne, und dies sowohl auf die Bühne, auf der Der Schwierige gespielt wird, als auch auf die Bühne des adligen Vorkriegswelttheaters mit seiner ganzen "redensartlichen Sprachlosigkeit". ${ }^{25}$ Eigentlich Bestandteil der Figurenrede Stanis, ist dieser Satz zugleich eine Regieanweisung für eine gestische Beglaubigungshandlung: Das verlobte Paar hat die Bühne ohne den öffentlichen Vollzug des "pantomimische[n] Ausdruck[s] für eine außergewöhnliche, gewissermaßen familiengeschichtliche Situation" (144), die öffentliche Umarmung, verlassen. Um diesen Mangel zu kompensieren, inszeniert Stani stattdessen eine Umarmung zwischen seiner Mutter, der Schwester des Bräutigams, und dem Vater der Braut: „Geh Sie, “weist Stani Crescence an, „sans mot dire auf ihn zu und umarm' Sie ihn, und das Ganze wird sein richtiges, offizielles Gesicht bekommen." (Ebd.) Damit schließt sich der Kreis der rekursiven Operationen, in denen sich die Sprachkritik des Schwierigen vollzieht: Die Dialoge handeln von der Unmöglichkeit verbindlichen Sprechens, die die Dialoge überschreitende Zeichenrede in Brücke, Schlüssel und Gesicht von der Unmöglichkeit, das Unmögliche sprachlich zu fassen; wird aber über Brücke, Schlüssel und Gesicht gesprochen, dann gelingt auf der Ebene der, kleinen Zeichen' sans mot dire', was auf der Ebene der groß ausgefalteten strategischtheatralischen Salonkonversation an Bühls sprachkritischem Bewusstsein von vornherein zum Scheitern verurteilt ist. ${ }^{26}$ Und so ist es auch nicht die Geste

\footnotetext{
23 Ebd., S. 290 f.

24 Berta Szeps-Zuckerkandl, Ich erlebte fünfzig Jahre Weltgeschichte, Stockholm, 1939, S. 264.

25 Arntzen, „Der Schwierige und der Nörgler", S. 35 o.

26 Vgl. dazu auch Hans Weigel, „Triumph der Wortlosigkeit: Ein Versuch über Hugo von Hofmannsthals Lustspiel, Der Schwierige "', in: ders., Nach wie vor Wörter, Graz, 1985, S. 298-313.
} 
von Crescences Umarmung des Brautvaters, mit der diese Form der poetologisch indirekten Kommunikation - und damit die poetische Sprache der Komödie insgesamt - ihr ,richtiges, offizielles Gesicht" erhält, sondern die Geste, mit der abschließend noch einmal darüber gesprochen wird, dass diese Sprache nur, sans mot dire' gesprochen werden kann. 